PROCEEDINGS OF THE

AMERICAN MATHEMATICAL SOCIETY

Volume 126, Number 2, February 1998, Pages 617-623

S 0002-9939(98)04284-1

\title{
ON SELF-ADJOINTNESS OF A SCHRÖDINGER OPERATOR ON DIFFERENTIAL FORMS
}

\author{
MAXIM BRAVERMAN
}

(Communicated by Jeffrey B. Rauch)

\begin{abstract}
Let $M$ be a complete Riemannian manifold and let $\Omega^{\bullet}(M)$ denote the space of differential forms on $M$. Let $d: \Omega^{\bullet}(M) \rightarrow \Omega^{\bullet+1}(M)$ be the exterior differential operator and let $\Delta=d d^{*}+d^{*} d$ be the Laplacian. We establish a sufficient condition for the Schrödinger operator $H=\Delta+V(x)$ (where the potential $V(x): \Omega^{\bullet}(M) \rightarrow \Omega^{\bullet}(M)$ is a zero order differential operator) to be self-adjoint. Our result generalizes a theorem by I. Oleinik about self-adjointness of a Schrödinger operator which acts on the space of scalar valued functions.
\end{abstract}

\section{INTRODUCTION}

Suppose $M$ is a complete Riemannian non-compact manifold. We will assume that $M$ is oriented and connected. Let $T^{*} M$ denote the cotangent bundle to $M$ and let $\wedge^{\bullet}\left(T^{*} M\right)=\bigoplus_{i} \wedge^{i}\left(T^{*} M\right)$ denote the exterior algebra of $T^{*} M$. We denote by $L^{2} \Omega^{\bullet}(M)$ the space of square integrable complex valued differential forms on $M$, i.e. the space of sections of $\bigwedge^{\bullet}\left(T^{*} M\right) \otimes \mathbb{C}$ which are square integrable with respect to the scalar product

$$
\langle\alpha, \beta\rangle=\int_{M} \alpha \wedge * \bar{\beta}, \quad \alpha, \beta \in L^{2} \Omega^{\bullet}(M) .
$$

Here $*$ denotes the Hodge operator associated to the Riemannian metric on $M$. Note that $L^{2} \Omega^{0}(M)$ is just the space of square integrable complex valued functions on $M$.

Let $d: L^{2} \Omega^{\bullet}(M) \rightarrow L^{2} \Omega^{\bullet+1}(M)$ denote the exterior differential and let $d^{*}$ be the operator formally adjoint to $d$ with respect to the scalar product (1).

Let $\Delta=d d^{*}+d^{*} d$ be the Laplacian and consider the Schrödinger operator

$$
H=\Delta+V(x): L^{2} \Omega^{\bullet}(M) \rightarrow L^{2} \Omega^{\bullet}(M)
$$

where the potential $V(x)$ is a measurable section of the bundle End $\left(\Lambda^{\bullet}\left(T^{*} M\right)\right)$ of endomorphisms of $\Lambda^{\bullet}\left(T^{*} M\right)$ which belongs to the class $L_{\text {loc }}^{\infty}$ (i.e. such that for any compact set $K \subset M$ there exists a constant $C_{K}>0$ such that $|V(x)| \leq C_{K}$ for almost all $x \in K)$.

Received by the editors August 19, 1996.

1991 Mathematics Subject Classification. Primary 58G25; Secondary 35P05.

The research was supported by US - Israel Binational Science Foundation grant No. 9400299.

(C)1998 American Mathematical Society 
We denote by $H_{0}$ the restriction of $H$ on the space $\Omega_{c}^{\bullet}(M)$ of smooth differential forms with compact support. The purpose of this paper is to introduce a sufficient condition on the potential $V(x)$ for operator $H_{0}$ to be self-adjoint.

\section{Statement of Results}

For $x, y \in M$ let $\operatorname{dist}(x, y)$ denote the Riemannian distance between $x$ and $y$. Fix a point $p \in M$ and set $r(x)=\operatorname{dist}(x, p)$.

Fix $x \in M$. The Riemannian metric on $M$ defines a scalar product $\langle\cdot, \cdot\rangle_{x}$ on the fiber $\Lambda^{\bullet}\left(T_{x}^{*} M\right) \otimes \mathbb{C}$ of the vector bundle $\Lambda^{\bullet}\left(T^{*} M\right) \otimes \mathbb{C}$. As usual, we write $V(x) \geq C$ if

$$
\langle V(x) \xi, \xi\rangle_{x} \geq C\langle\xi, \xi\rangle_{x}
$$

for any $\xi \in \Lambda^{\bullet}\left(T_{x}^{*} M\right) \otimes \mathbb{C}$. Note that it follows from (3) that $V(x)$ is a self-adjoint endomorphism of $\wedge^{\bullet}\left(T_{x}^{*} M\right)$.

Theorem A. Assume that for almost all $x \in M$ the potential $V(x)$ of the operator (2) satisfies the estimate

$$
V(x) \geq-Q(x),
$$

where $1 \leq Q(x) \leq \infty$ and $Q^{-1 / 2}(x)$ is a Lipschitz function on $M$ such that

$$
\left|Q^{-1 / 2}(x)-Q^{-1 / 2}(y)\right| \leq K \operatorname{dist}(x, y) \quad \text { for any } \quad x, y \in M \text {. }
$$

If for any piecewise smooth curve $\gamma:[0, \infty) \rightarrow M$ such that $\lim _{t \rightarrow \infty} r(\gamma(t))=\infty$ the integral

$$
\int_{\gamma} Q^{-1 / 2}(x) d \gamma=\infty
$$

then the operator $H_{0}$ is essentially self-adjoint.

For the case of a Schrödinger operator acting on scalar valued functions this theorem was established by I. Oleinik [O2]. Note that $Q(x)$ may be equal to infinity on a set of positive measure.

As a simple consequence of Theorem A we obtain the following

Theorem B. Suppose that for almost all $x \in M$ the potential $V(x)$ satisfies the estimate $V(x) \geq-q(r(x))$, where $1 \leq q \leq \infty$ and $q^{-1 / 2}(t)$ is a Lipschitz function on $\mathbb{R}$ such that $\int_{0}^{\infty} q^{-1 / 2}(t) d t=\infty$. Then the operator $H_{0}$ is essentially self-adjoint.

In particular, if $M=\mathbb{R}^{n}$ and $V(x) \geq-C|x|^{2}$ then the operator $H_{0}$ is essentially self-adjoint.

Remark. Theorem A remains true if we replace $L^{2} \Omega^{\bullet}(M)$ by the space of square integrable forms on $M$ with values in a flat Hermitian vector bundle $\mathcal{F}$ over $M$, provided that the Hermitian structure on $\mathcal{F}$ is flat. In this case the differential $d$ should be replaced by the covariant differential associated to the flat structure on $\mathcal{F}$. The proof is a verbatim repetition of the proof for the scalar case, cf. below. However the notation in the vector valued case is more complicated. 


\section{HistORICAL REMARKS}

An analogue of Theorem B for the case $M=\mathbb{R}^{1}$ was established by Sears [Se]. B. Levitan [Le] proved the Sears theorem for the Schrödinger operator acting on scalar valued functions on $M=\mathbb{R}^{n}$. F. Rofe-Beketov [RB] extended these results to the case where the potential $V(x)$ cannot be estimated by a function depending only on $\operatorname{dist}(x, p)$. Many results and references about the essential self-adjointness of Schrödinger operators on $\mathbb{R}^{n}$ may be found in [RS].

I. Oleinik [O1], [O2] established Theorem A for the Schrödinger operator acting on scalar valued functions on a complete Riemannian manifold.

Essential self-adjointness of a pure Laplacian (without lower order terms) on differential forms on a complete Riemannian manifold was first stated and proved by M. P. Gaffney [Ga1], [Ga2]. A much more general statement was proven by P. Chernoff $[\mathrm{Ch}]$. A number of related results may be found in [Sh].

\section{The domain of $\mathcal{D}\left(H_{0}^{*}\right)$}

Let $H_{0}^{*}$ denote the operator adjoint to $H_{0}$. The domain $\mathcal{D}\left(H_{0}^{*}\right)$ of $H_{0}^{*}$ consists of forms $\alpha \in L^{2} \Omega^{\bullet}(M)$ such that $H \alpha$ understood in the sense of distributions also belongs to $L^{2} \Omega^{\bullet}(M)$.

The operator $H_{0}$ is symmetric. Hence, to show that its closure is self-adjoint it is enough to show that the adjoint operator $H_{0}^{*}$ is symmetric. In other words we have to prove that

$$
\int_{M}(H \alpha \wedge * \bar{\beta}-\alpha \wedge * \overline{H \beta})=0 \quad \text { for any } \alpha, \beta \in \mathcal{D}\left(H_{0}^{*}\right) .
$$

To prove (7) we need some information about the behavior of differential forms from $\mathcal{D}\left(H_{0}^{*}\right)$. The main result of this section is the following lemma, which provides us with this information.

Lemma 1. If $\alpha \in \mathcal{D}\left(H_{0}^{*}\right)$ then the forms $Q^{-1 / 2} d \alpha, Q^{-1 / 2} d^{*} \alpha$ are square integrable.

Remark. 1. By the standard theory of elliptic operators any $\alpha \in \mathcal{D}\left(H_{0}^{*}\right)$ belongs to the Sobolev space $H_{l o c}^{2}$. Hence, $d \alpha, d^{*} \alpha$ are locally square integrable. Thus the lemma provides us with information about the behavior of the forms from $\mathcal{D}\left(H_{0}^{*}\right)$ at infinity.

2. For the Schrödinger operator on scalar valued functions on $\mathbb{R}^{n}$ an analogous lemma was established in [RB]. The proof was adopted in [O1], [O2] to the case of a Riemannian manifold. In our proof we follow rather closely the lines of [O2]. However, the fact that we deal with differential forms rather than with scalar valued functions demands a more careful analysis.

Proof. Recall that we fixed a point $p \in M$ and that for any $x \in M$ we denoted by $r(x)$ the Riemannian distance between $x$ and $p$.

It is shown in [O2, Proof of Lemma 1] that for any $R>0, \varepsilon>0$ there exist smooth functions $r_{R, \varepsilon}(x), F_{R, \varepsilon}(x)$ on $M$ which approximate the Lipschitz functions $r(x), Q^{-1 / 2}(x)$ in the sense that

$$
\begin{gathered}
\left|r_{R, \varepsilon}(x)-r(x)\right|<\varepsilon, \quad Q^{-1 / 2}(x)-\varepsilon<F_{R, \varepsilon}(x)<(1+\varepsilon) Q^{-1 / 2}(x), \\
\varlimsup_{\varepsilon \rightarrow 0}\left|d r_{R, \varepsilon}(x)\right| \leq 1, \quad \varlimsup_{\varepsilon \rightarrow 0}\left|d F_{R, \varepsilon}(x)\right| \leq K,
\end{gathered}
$$

for any $x \in r_{R, \varepsilon}^{-1}([0, R+1])$. Here $K$ is the same constant as in (5). 
Let $\Psi:[0,+\infty) \rightarrow[0,1]$ be a smooth function which is equal to one when $t \leq 1 / 2$ and which is equal to zero when $t \geq 1$. Set

$$
\psi_{R, \varepsilon}(x)= \begin{cases}\Psi\left(\frac{r_{R, \varepsilon}(x)}{R}\right) F_{R, \varepsilon}(x) & \text { if } r_{R, \varepsilon}(x) \leq R \\ 0 & \text { outside of the set } r_{R, \varepsilon}(x) \leq R .\end{cases}
$$

For any $R>0$ the functions $\psi_{R, \varepsilon}, \varepsilon<1$ vanish outside of the compact set $r^{-1}([0, R+1])$. Hence, it follows from (8) and (4) that there exist a constant $K_{1}>0$ not depending on $R$ and a number $\varepsilon_{R}>0$ (which does depend on $R$ ) such that

$$
\left|d \psi_{R, \varepsilon}(x)\right| \leq K_{1}, \quad \psi_{R, \varepsilon}^{2}(x) \leq 2, \quad\left|\int_{M} \psi_{R, \varepsilon}^{2} \alpha \wedge * V \alpha\right| \leq 2\|\alpha\|^{2},
$$

for any $x \in M, R>1,0<\varepsilon<\varepsilon_{R}, \alpha \in L^{2} \Omega^{\bullet}(M)$. Here $\|\alpha\|=\langle\alpha, \alpha\rangle^{\frac{1}{2}}$ denotes the $L^{2}$-norm of the form $\alpha$.

Functions $\psi_{R, \varepsilon}$ have compact support. Hence, in view of Remark 1 after the statement of the lemma, the forms $\psi_{R, \varepsilon} d \alpha$ and $\psi_{R, \varepsilon} d^{*} \alpha$ are square integrable. Assume that $\alpha \in \mathcal{D}\left(H_{0}^{*}\right)$ is a real valued form and set

$$
J_{R, \varepsilon}^{2}=\left\|\psi_{R, \varepsilon} d \alpha\right\|^{2}+\left\|\psi_{R, \varepsilon} d^{*} \alpha\right\|^{2}=\int_{M} \psi_{R, \varepsilon}(x)^{2}\left(d \alpha \wedge * d \alpha+d^{*} \alpha \wedge * d^{*} \alpha\right) .
$$

It follows from (8), (9) that to prove the lemma it is enough to show that

$$
\varlimsup_{R \rightarrow \infty} \varlimsup_{\varepsilon \rightarrow 0} J_{R, \varepsilon}<\infty .
$$

Let us first rewrite the integrand in (11) in a more convenient form. In the calculations bellow we use the equality (cf. [Wa, §6.1]) $d^{*} \alpha=(-1)^{|\alpha|} *^{-1} d * \alpha$ where $|\alpha|$ denotes the degree of the differential form $\alpha$.

$$
\psi_{R, \varepsilon}^{2} d \alpha \wedge * d \alpha=d\left(\psi_{R, \varepsilon}^{2} \alpha \wedge * d \alpha\right)-2 \psi_{R, \varepsilon} d \psi_{R, \varepsilon} \wedge \alpha \wedge * d \alpha+\psi_{R, \varepsilon}^{2} \alpha \wedge * d^{*} d \alpha,
$$

$$
\begin{aligned}
& \psi_{R, \varepsilon}^{2} d^{*} \alpha \wedge * d^{*} \alpha=(-1)^{|\alpha|} \psi_{R, \varepsilon}^{2} d^{*} \alpha \wedge d * \alpha \\
& =-d\left(\psi_{R, \varepsilon}^{2} d^{*} \alpha \wedge * \alpha\right)+2 \psi_{R, \varepsilon} d \psi_{R, \varepsilon} \wedge d^{*} \alpha \wedge * \alpha+\psi_{R, \varepsilon}^{2} d d^{*} \alpha \wedge * \alpha .
\end{aligned}
$$

It follows now from (13), (10) and from the Stokes theorem that, if $R>1, \varepsilon<\varepsilon_{R}$, then

$$
\begin{aligned}
\left\|\psi_{R, \varepsilon} d \alpha\right\|^{2} & =\int_{M} \psi_{R, \varepsilon}^{2} d \alpha \wedge * d \alpha=-2\left\langle d \psi_{R, \varepsilon} \wedge \alpha, \psi_{R, \varepsilon} d \alpha\right\rangle+\left\langle\alpha, \psi_{R, \varepsilon}^{2} d^{*} d \alpha\right\rangle \\
& \leq 2 K_{1}\|\alpha\|\left\|\psi_{R, \varepsilon} d \alpha\right\|+\left\langle\alpha, \psi_{R, \varepsilon}^{2} d^{*} d \alpha\right\rangle \\
\left\|\psi_{R, \varepsilon} d^{*} \alpha\right\|^{2} & =\int_{M} \psi_{R, \varepsilon}^{2} d^{*} \alpha \wedge * d^{*} \alpha=2\left\langle d \psi_{R, \varepsilon} \wedge \psi_{R, \varepsilon} d^{*} \alpha, \alpha\right\rangle+\left\langle\psi_{R, \varepsilon}^{2} d d^{*} \alpha, \alpha\right\rangle \\
& \leq 2 K_{1}\left\|\psi_{R, \varepsilon} d^{*} \alpha\right\|\|\alpha\|+\left\langle\alpha, \psi_{R, \varepsilon}^{2} d d^{*} \alpha\right\rangle .
\end{aligned}
$$

Summing these two equations we obtain

$$
\begin{aligned}
J_{R, \varepsilon}^{2} & \leq 2 K_{1}\|\alpha\|\left(\left\|\psi_{R, \varepsilon} d \alpha\right\|+\left\|\psi_{R, \varepsilon} d^{*} \alpha\right\|\right)+\left\langle\alpha, \psi_{R, \varepsilon}^{2} \Delta \alpha\right\rangle \\
& \leq 4 K_{1}\|\alpha\| J_{R, \varepsilon}+\int_{M} \psi_{R, \varepsilon}^{2}(\alpha \wedge * H \alpha-\alpha \wedge * V \alpha) \\
& \leq 4 K_{1}\|\alpha\| J_{R, \varepsilon}+2\|\alpha\|\|H \alpha\|+2\|\alpha\|^{2} .
\end{aligned}
$$

Here the last inequality follows from (10). 
It follows from (14) that the set $\left\{J_{R, \varepsilon}: R>1, \varepsilon<\varepsilon_{R}\right\}$ is bounded from above. Hence (12) holds. The proof of the lemma is completed.

\section{Proof of Theorem A}

We apply a modification of the method used in [RB] suggested by I. Oleinik [O2]. The quantity

$$
\widetilde{\rho}(x, y)=\inf _{\gamma} \int_{\gamma} Q^{-1 / 2}(x) d \gamma,
$$

where the infimum is taken over all piecewise smooth curves connecting the points $x, y \in M$, is called generalized distance between $x$ and $y$. It is a symmetric function in $x, y$ which satisfies the triangular inequality. The first metric axiom is not valid in general. Note, however, that (6) implies that the sets $P^{-1}([0, R])$ are compact for any $R>0$.

Recall that in Section 2 we have fixed a point $p \in M$. Set $P(x)=\widetilde{\rho}(x, p)$. Then (cf. [O2, Lemma 2])

$$
|P(x)-P(y)| \leq Q^{-1 / 2}(x) \operatorname{dist}(x, y)+\frac{K}{2}(\operatorname{dist}(x, y))^{2}
$$

for any $x, y \in M$. It follows (cf. [O2]) that for any $R>0, \varepsilon>0$ there exists a smooth function $\widetilde{P}_{R, \varepsilon}(x)$ which approximates $P(x)$ in the sense that

$$
\left|\widetilde{P}_{R, \varepsilon}(x)-P(x)\right| \leq \varepsilon, \quad \varlimsup_{\varepsilon \rightarrow 0}\left|d \widetilde{P}_{R, \varepsilon}(x)\right| \leq Q^{-1 / 2}(x),
$$

for any $x \in P^{-1}([0, R+1])$.

Assume that $\varepsilon<1$ so that $\widetilde{P}_{R, \varepsilon}^{-1}([0, R]) \subset P^{-1}([0, R+1])$. Let us define a piecewise smooth function $P_{R, \varepsilon}(x)$ on $M$ by the formula

$$
P_{R, \varepsilon}(x)= \begin{cases}\widetilde{P}_{R, \varepsilon}(x) & \text { if } \widetilde{P}_{R, \varepsilon}(x) \leq R \\ R & \text { outside the set } \widetilde{P}_{R, \varepsilon}(x) \leq R .\end{cases}
$$

By (17), the inequality

$$
\varlimsup_{\varepsilon \rightarrow 0}\left|d P_{R, \varepsilon}(x)\right| \leq Q^{-1 / 2}(x)
$$

holds almost everywhere on $M$.

Recall from Section 4 that the statement of Theorem A is equivalent to equality (7). Fix $\alpha, \beta \in \mathcal{D}\left(H_{0}^{*}\right)$ and consider the following approximation of the integral (7):

$$
\begin{aligned}
I_{R, \varepsilon} & =\int_{M}\left(1-\frac{P_{R, \varepsilon}}{R}\right)(H \alpha \wedge * \bar{\beta}-\alpha \wedge * \overline{H \beta}) \\
& =\int_{M}\left(1-\frac{P_{R, \varepsilon}}{R}\right)(\Delta \alpha \wedge * \bar{\beta}-\alpha \wedge * \overline{\Delta \beta}) .
\end{aligned}
$$

By the Fatou theorem ([RS, Theorem I.17]), it is enough to show that

$$
\varlimsup_{R \rightarrow \infty} \varlimsup_{\varepsilon \rightarrow 0} I_{R, \varepsilon}=0 .
$$

We will need the following "integration by parts" lemma. ${ }^{1}$

\footnotetext{
${ }^{1}$ I learned this lemma from M. Shubin.
} 
Lemma 2. Let $\phi: M \rightarrow \mathbb{R}$ be a smooth function with compact support. Then

$$
\begin{aligned}
\int_{M} \phi & \Delta \alpha \wedge * \bar{\beta} \\
& =\int_{M} \phi\left(d \alpha \wedge * d \bar{\beta}+d^{*} \alpha \wedge * d^{*} \bar{\beta}\right)+\int_{M} d \phi \wedge\left(\bar{\beta} \wedge * d \alpha-d^{*} \alpha \wedge * \bar{\beta}\right)
\end{aligned}
$$

for any $\alpha, \beta \in \mathcal{D}\left(H_{0}^{*}\right)$.

Note that, by Remark 1 after the statement of Lemma 1, all the integrals in (22) make sense.

Proof. Recall that $d^{*} u=(-1)^{|u|} *^{-1} d * u$ where $|u|$ denotes the degree of the differential form $u$. Hence, if $|u|=|v|-1$, then

$$
\phi d u \wedge * v=\phi u \wedge * d^{*} v-d \phi \wedge u \wedge * w+d(\phi u \wedge * v) .
$$

Substituting into (23) first $u=d^{*} \alpha, v=\bar{\beta}$ and then $u=\bar{\beta}, v=d \alpha$ we obtain

$$
\begin{aligned}
& \phi d d^{*} \alpha \wedge * \bar{\beta}=-d \phi \wedge d^{*} \alpha \wedge * \bar{\beta}+\phi d^{*} \alpha \wedge * d^{*} \bar{\beta}+d\left(\phi d^{*} \alpha \wedge * \bar{\beta}\right), \\
& \phi d^{*} d \alpha \wedge * \bar{\beta}=\phi \bar{\beta} \wedge * d^{*} d \alpha=d \phi \wedge \bar{\beta} \wedge d \alpha+\phi d \alpha \wedge * d \bar{\beta}-d(\phi \bar{\beta} \wedge * d \alpha) .
\end{aligned}
$$

In the last equality we used that $u \wedge * v=v \wedge * u$ for any differential forms $u, v$ of the same degree. Summing the above equations, integrating over $M$ and using the Stokes theorem we get (22).

Using definition (20) of $I_{R, \varepsilon}$ and Lemma 2 we obtain

$$
I_{R, \varepsilon}=\frac{1}{R} \int_{M} d P_{R, \varepsilon} \wedge\left(\bar{\beta} \wedge * d \alpha-d^{*} \alpha \wedge * \bar{\beta}-\alpha \wedge * d \bar{\beta}+d^{*} \bar{\beta} \wedge * \alpha\right) .
$$

Let $d \mu(x)$ denote the Riemannian density on $M$. For any $\xi \in \bigwedge^{k}\left(T^{*} M\right) \otimes \mathbb{C}$ we denote by $|\xi|$ its norm with respect to the scalar product on $\Lambda^{\bullet}\left(T^{*} M\right) \otimes \mathbb{C}$ induced by the Riemannian structure on $M$. Then

$$
|\langle\alpha, \beta\rangle| \leq \int_{M}|\alpha \wedge * \bar{\beta}| d \mu(x) \leq \int_{M}|\alpha||\beta| d \mu(x) \leq\|\alpha\|\|\beta\|
$$

for any $\alpha, \beta \in L^{2} \Omega^{\bullet}(M)$.

Let us estimate the behavior of the right-hand side of (24) as $\varepsilon \rightarrow 0$. For the first term we obtain

$$
\begin{gathered}
\varlimsup_{\varepsilon \rightarrow 0}\left|\frac{1}{R} \int_{M} d P_{R, \varepsilon} \wedge \bar{\beta} \wedge * d \alpha\right| \leq \frac{1}{R} \varlimsup_{\varepsilon \rightarrow 0} \int_{M}\left|d P_{R, \varepsilon}\right|\left|d^{*} \alpha\right||\bar{\beta}| d \mu(x) \\
\leq \frac{1}{R} \int_{M}\left|Q^{-1 / 2} d^{*} \alpha\right||\bar{\beta}| d \mu(x) \leq \frac{\left\|Q^{-1 / 2} d^{*} \alpha\right\|\|\beta\|}{R} .
\end{gathered}
$$

In the second inequality in (26) we used the estimate (19). The last inequality in (26) follows from Lemma 1.

Analogously, one can estimate the other terms in the right-hand side of (24). That proves (21) and Theorem A.

\section{ACKNOWLEDGMENT}

I would like to thank M. Shubin for posing the problem and for essential help.

I am very grateful to I. Oleinik for pointing out a gap in a preliminary version of the paper and for drawing my attention to the paper [O2].

I am also thankful to M. Farber and V. Matsaev for valuable discussions. 


\section{REFERENCES}

[BS] F. A. Berezin, M. A. Shubin, The Schrödinger equation, Kluwer, Dordrecht, 1991. MR 93i: 81001

[Ch] P. R. Chernoff, Essential self-adjointness of powers of generators of hyperbolic equations, J. of Functional Analysis 12 (1973), 401-414. MR 51:6119

[Ga1] M. P. Gaffney, The harmonic operators for exterior differential forms, Proc. Nat. Acad. Sci. USA 37 (1951), 48-50. MR 13:987b

[Ga2] M. P. Gaffney, A special Stokes's theorem for complete Riemannian manifolds, Ann. of Math. 60 (1954), 140-145. MR 15:986d

[Le] B. M. Levitan, On a theorem of Titchmarsh and Sears, Usp. Math. Nauk 16 (1961), 175-178. MR 24:A2133

[O1] I. M. Oleinik, On the essential self-adjointness of the Schrödinger operator on a complete Riemannian manifold, Mathematical Notes 54 (1993), 934- 939. MR 94m:58226

[O2] I. M. Oleinik, On the connection of the classical and quantum mechanical completeness of a potential at infinity on complete Riemannian manifolds, Mathematical Notes 55 (1994), 380-386. MR 95h:35051

[RB] F. S. Rofe-Beketov, Self-adjointness conditions for the Schrödinger operator, Mat. Zametki 8 (1970), 741-751. MR 43:743

[RS] M. Reed, B. Simon, Methods of modern mathematical physics, Vol. I, II, Academic Press, London, 1972, 1975. MR 58:12429a; MR 58:12429b

[Se] D. B. Sears, Note on the uniqueness of Green's functions associated with certain differential equations, Canadian J. Math. 2 (1950), 314-325. MR 12:102h

[Sh] M. A. Shubin, Spectral theory of elliptic operators on non-compact manifolds, Astérisque 207 (1992), 37-108. MR 94h:58175

[Wa] F. W. Warner, Foundations of differentiable manifolds and Lie groups, Graduate Texts in Mathematics, Springer-Verlag, New-York, Berlin, Heidelberg, Tokyo, 1983. MR 84k:58001

Department of Mathematics, Ohio State University, Columbus, Ohio 43210

E-mail address: maxim@math.ohio-state.edu

Current address: Department of Mathematics, The Hebrew University, Jerusalem 91904, Israel 